\title{
Systematic assessment of dexmedetomidine as an anesthetic agent: a meta-analysis of randomized controlled trials
}

\author{
Guanying Piao ${ }^{1,2}$, Jiarui $\mathrm{Wu}^{1,2}$
}

${ }^{1}$ School of Life Sciences, University of Science and Technology of China, Hefei, China ${ }^{2}$ Key Laboratory of Systems Biology, SIBS-Novo Nordisk Translational Research Centre for PreDiabetes, Shanghai Institutes for Biological Sciences, Chinese Academy of Sciences, Shanghai, China

Submitted: 6 March 2013

Accepted: 4 June 2013

Arch Med Sci 2014; 10, 1: 19-24

DOI: 10.5114/aoms.2014.40730

Copyright @ 2014 Termedia \& Banach

\section{Abstract}

Introduction: Here we aimed to study the effectiveness of dexmedetomidine as an anesthetic adjunct in surgery.

Material and methods: A systematic evaluation was performed on published clinical trials. Major databases such as Medline database were employed to search and identify relevant studies and then Rev.Man 5 was used for meta-analysis as well as forest plots. Mean difference (MD) was chosen as the effect size for measurement data, while odds ratio (OR) was calculated for enumeration data.

Results: A total of 18 studies met the inclusion criteria. The postoperative heart rate and mean arterial pressure for the dexmedetomidine group were significantly lower than the control group (combined MDs were -14.12 and -9.96). The incidence rates of postoperative nausea and vomiting, chills, and shivering of the dexmedetomidine group were lower than the control group (pooled ORs were $0.41,0.21$ and 0.14 , respectively). However, the occurrence rates of bradycardia and hypotension in the dexmedetomidine group were higher than the control group (pooled ORs were 5.14 and 3.00).

Conclusions: Dexmedetomidine can stabilize blood pressure and heart rate, and prevent postoperative adverse reactions. However, patients with original hypovolemia or heart block should be cautious. Besides, the quality of such studies should be improved in methodology to evaluate their efficacy and safety comprehensively.

Key words: dexmedetomidine, anesthesia, sedation, systematic evaluation.

\section{Introduction}

Surgery induces a stress response that may result in prolonged postoperative convalescence [1]. Thus anesthetic management is necessary to modulate this physiological response. Dexmedetomidine is one of the $\alpha 2$ adrenergic receptor agonists, which have been used as antihypertensive drugs in some clinical trials. In recent years, studies indicated that $\alpha 2$ receptor agonists possess sedative properties and thus some of them are used as adjuvant drugs in anesthesia. Dexmedetomidine has high selectivity and good potency and thus has drawn a lot of attention in the United States [2]. It came into common usage in 2004 in Japan [3, 4] and

\author{
Corresponding author: \\ Guanying Piao \\ School of Life Sciences \\ University of Science \\ and Technology of China \\ Hefei 230026, China \\ Phone: +860551 63602469 \\ E-mail: \\ piaogy@mail.ustc.edu.cn
}


the clinical trials and application of dexmedetomidine began in China in $2007[5,6]$.

Dexmedetomidine mainly acts on $\alpha 2$ receptors in the brain and spinal cord. It affects the functions of central nervous, circulatory and respiratory systems and exhibits sympatholytic, sedative and analgesic properties [7] through the following mechanisms: inhibition of protein kinase $A$ and subsequent phosphorylation, activation of potassium channels and hyperpolarization of plasma membrane, inhibition of adenylate cyclase activity, reduction of neuronal firing as well as inhibition of voltage-gated calcium ion channels [8].

Though dexmedetomidine possesses good properties, it often produces hypotension and bradycardia [9]. It is necessary to carry out a systematic evaluation as it is widely used in anesthesia. Several studies have provided evaluations with focus on its intensive care unit (ICU) application [10] and cardiac protection [11]. Thus we performed a meta-analysis of general randomized controlled trials to understand its effectiveness and safety and provide guidance for its clinical applications.

\section{Material and methods}

\section{Inclusion, exclusion criteria and retrieval strategy}

Inclusion criteria: Randomized controlled trials (RCTs) researching the efficacy and safety of dexmedetomidine in general anesthesia were included. Dexmedetomidine was used as an adjunct drug for sedation and analgesia in the experimental group, while saline was used as a placebo in the control group. Dexmedetomidine or placebo was given during the anesthesia induction phase or maintenance phase. Other drugs were kept consistent between the two groups. Additionally, the following outcome measures should be contained in the studies: (1) the hemodynamic parameters: mean arterial pressure and heart rate at the end of surgery (usually within $3 \mathrm{~h}$ after surgery); (2) indicators of postoperative adverse reactions, such as nausea and vomiting, chills and shivering; (3) safety (or side effects) indicators such as perioperative hypotension and bradycardia.

Exclusion criteria: (1) dexmedetomidine was used in the postoperative ICU care; (2) although curves were provided for indicators, no specific data could be extracted; (3) a variety of sedatives were applied, not only dexmedetomidine; (4) multi-group trials and quasi-randomized controlled trials.

Retrieval method: MEDLINE, EMBASE, EBSCO, Springer, Ovid and Cochrane library were searched with the keywords dexmedetomidine and randomized controlled trials. The deadline was July 2012.

\section{Quality assessment and data extraction}

Quality assessment was performed in the following aspects: (1) the random allocation method adopted; (2) whether in a blind fashion; (3) evaluation on exit of trial.

The following information were extracted: (1) general information, such as the subject of the study, name of the researchers and source of the literature; (2) characteristics of the study: experimental design, research measures, implementation methods, measures to prevent bias, main results, etc.; (3) outcome measures: number of case for each group, statistical number for all measurement data, and number of case for all measurement data.

\section{Statistical analysis}

Rev.Man 5 provided by the Cochrane Collaboration was used for meta-analysis and forest plot. Mean difference (MD) was calculated as the effect size for measurement data and odds ratio (OR) for enumeration data. The 95\% confidence interval $(\mathrm{Cl})$ was also provided. The chi-square $\left(\chi^{2}\right)$ test was performed to determine the heterogeneity among studies. If homogeneity existed $(p>0.05)$, a fixed-effects model was used in analysis. If not $(p<0.05)$, a random-effects model was used.

\section{Results}

\section{General information}

A total of 18 studies [12-29] published during 1992-2012 met the criteria and were included in the analysis. Sixteen of them adopted a double-blind design while the other two did not [26, 27]. Six studies did not describe the randomization method [23, 25-29]. Major maintaining narcotics were sevoflurane, isoflurane, propofol and fentanyl. The dose of dexmedetomidine was between $0.5 \mu \mathrm{g} / \mathrm{kg}$ and $2.5 \mu \mathrm{g} / \mathrm{kg}$.

\section{Efficacy qnalysis}

\section{Cardiac rate}

Cardiac rate was reported in 5 studies. As between-study heterogeneity existed $(p<0.05)$, a random-effects model was adopted. The combined MD was $-14.12(95 \% \mathrm{Cl}:-18.02,-10.21)$ and it was significant $(Z=7.09, p<0.00001)$. Thus the heart rate of the dexmedetomidine group was significantly lower compared with the control group (Figure 1).

\section{Mean arterial blood pressure}

A total of 4 studies analyzed the influence of dexmedetomidine on mean arterial blood pressure. No between-study heterogeneity existed ac- 


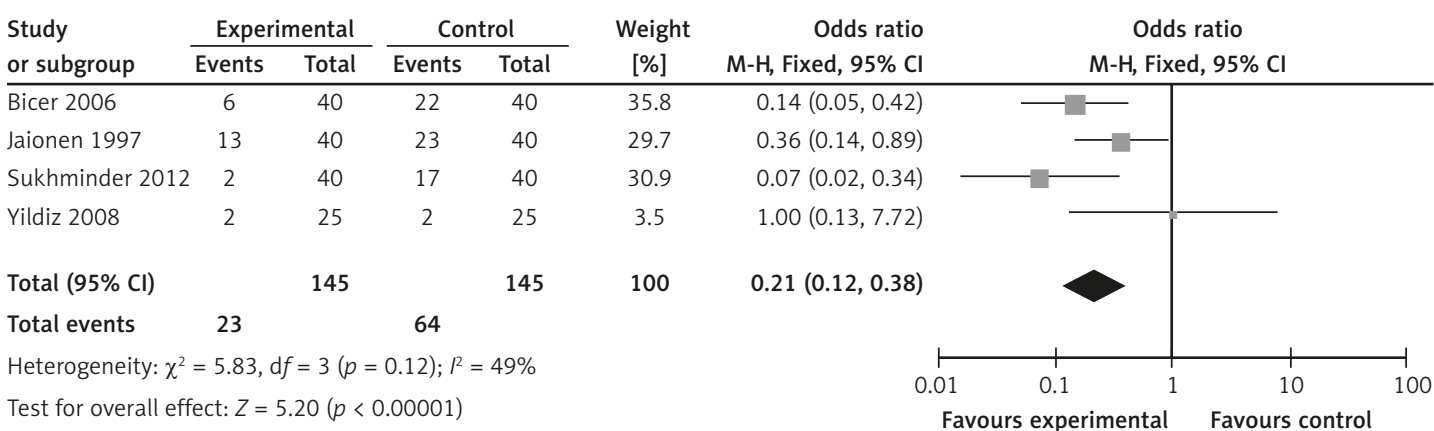

Figure 4. Statistical results for incidence of chill from 4 studies

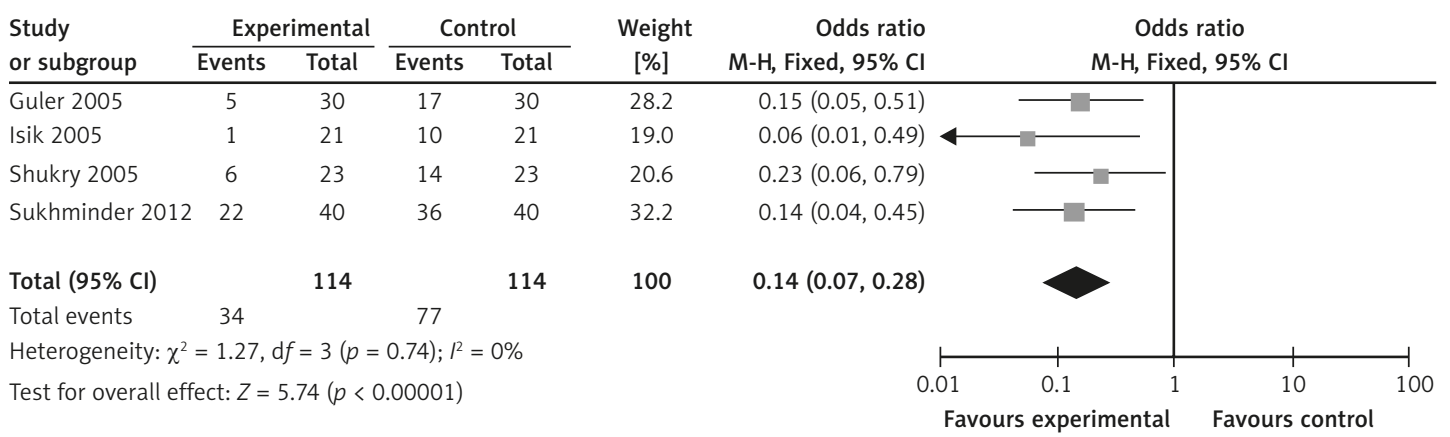

Figure 5. Statistical results for incidence of restlessness from 4 studies

\section{Incidence of restlessness}

A total of 4 studies provided information regarding restlessness. As there was no heterogeneity, a fixed-effects model was utilized to determine the pooled OR as $0.14(95 \% \mathrm{Cl}: 0.07,0.28)$. Statistical analysis showed that it was significant ( $Z=5.74, p<0.00001)$, so it can be concluded that dexmedetomidine significantly reduced the incidence of restlessness in comparison with the control (Figure 5).

\section{Incidence of bradycardia}

Incidence of bradycardia was reported in 7 studies. Since no between-study heterogeneity was observed $(p>0.05)$, a fixed-effects model was used.
The pooled OR was $5.14(95 \% \mathrm{Cl}: 2.25,11.76)$ and it was significant $(Z=3.38, p=0.0001)$, which indicated that dexmedetomidine resulted in a significantly higher incidence of bradycardia compared with the control (Figure 6).

\section{Incidence of hypotension}

Incidence of hypotension was extracted from 6 studies. As there was no between-study heterogeneity, a fixed-effects model was adopted. The pooled OR was calculated as $3.00(95 \% \mathrm{Cl}: 1.21$, $7.45)$ and it was significant $(Z=2.37, p=0.02)$. Thus dexmedetomidine resulted in a significantly higher incidence of hypotension in comparison with the control (Figure 7).

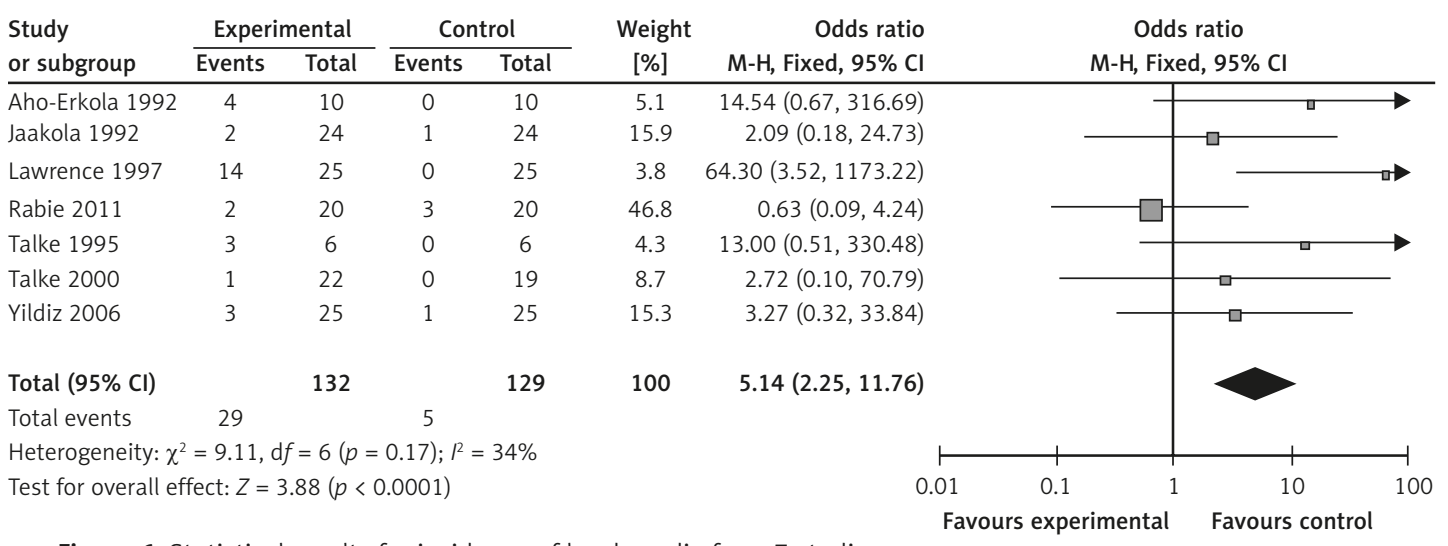

Figure 6. Statistical results for incidence of bradycardia from 7 studies 


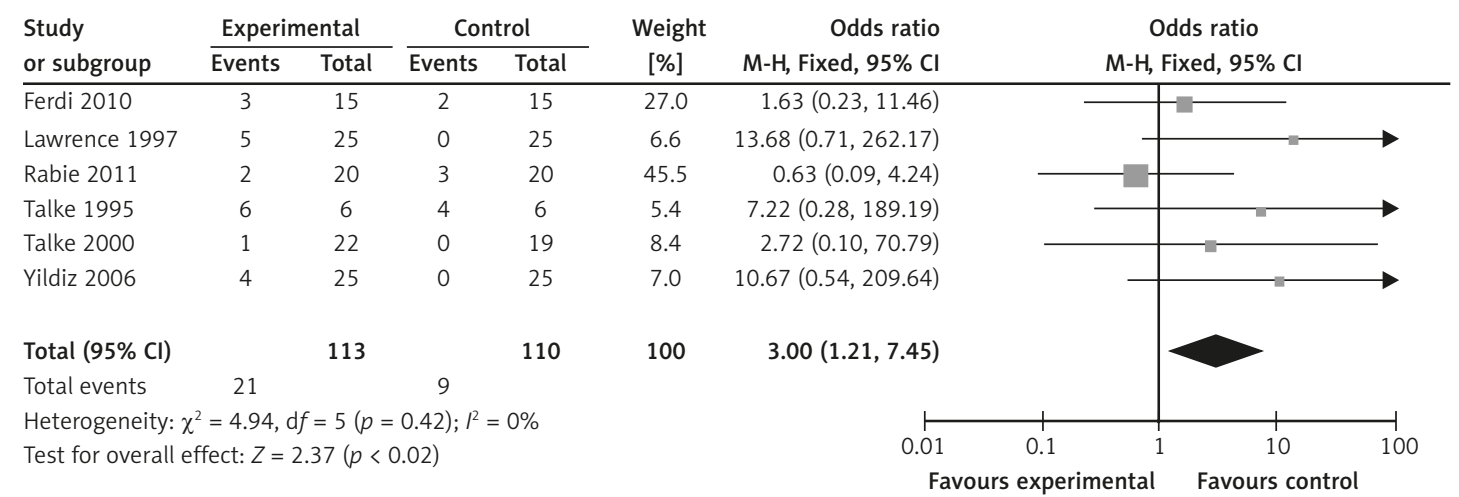

Figure 7. Statistical results for incidence of hypotension from 6 studies

\section{Discussion}

In general anesthesia, patients generally produce a stress response to the cardiovascular system because of invasive damage due to surgical instruments. If the stability of the cardiovascular system is destroyed, fluctuation in blood pressure and heart rate will occur, which may further lead to hypertension and tachycardia. Dexmedetomidine is a highly selective $\alpha 2$ receptor agonist that can reduce the sensitivity of the sympathetic nervous system and maintain the stability of cardiovascular function in patients with surgery. A previous study showed that pre-operative intravenous administered dexmedetomidine could attenuate the increase of norepinephrine caused by tracheal intubation and surgery [30]. Another study showed that dexmedetomidine lowered peripheral vascular resistance and stabled the blood flow velocity during the operation [31]. However, due to its short half-life and high elimination speed, we are uncertain whether the effect of dexmedetomidine can be maintained to the end of surgery when it is injected within the usual safe dose range $(0.5-2.5 \mu \mathrm{g} / \mathrm{kg})$. We analyzed postoperative heart rate and mean arterial pressure and found that they were significantly lower in the dexmedetomidine group than the placebo group. Therefore, it can be concluded that the hemodynamic effects of dexmedetomidine can be maintained to the end of the surgery.

Postoperative nausea and vomiting, chills and restlessness are common adverse reactions in anesthesia surgery. Nausea and vomiting not only decrease the patients' life quality, but also slow down the speed of recovery and even prolong hospitalization. Postoperative restlessness affects the wound healing process. Chills will raise intracranial pressure, lead to cardiovascular adverse reactions and increase wound pain. Dexmedetomidine inhibits the central nervous system by acting on the locus coeruleus and controlling downstream the medulla oblongata. Some studies indicated that dexmedetomidine significantly reduced the occurrence of postoperative nausea and vomiting
[19], and also decreased the incidence of agitation [12]. It was also found that dexmedetomidine could significantly lower the incidence of shivering and its effect was even better than midazolam [32]. This study reached the same conclusion after a systematic evaluation showing that dexmedetomidine had a significant effect on the incidence of postoperative nausea and vomiting, chills as well as restlessness.

Dexmedetomidine can lead to a decline in blood pressure and heart rate and thus has a good effect in the control of the surgical stress response. However, dexmedetomidine will cause hypotension and bradycardia, which presents certain risks to patients with original low blood volume or heart block. We analyzed the occurrence of perioperative hypotension and bradycardia, and found a significantly higher incidence in patients with dexmedetomidine. It is necessary for anesthesiologists to add appropriate atropine and other drugs during the operation to alleviate the condition.

Our meta-analysis contains some limitations. First, the publication bias was not assessed using a funnel plot due to the small number of studies $(<10)$ included in the analyses of the outcome measures. Second, the randomization method was not described in some papers and the double-blind method was not adopted in several studies, so it is necessary to be cautious in the interpretation of these research results. Third, hemodynamic indicators are affected by the types of surgery and thus cannot be merged to carry out a systematic evaluation. Subsequent studies should strictly control the experimental conditions to better address these questions. Finally, chills and restlessness lack specific measurement methods, which need to be developed in the future to distinguish different levels and thus help to better understand the effectiveness of dexmedetomidine.

\section{Acknowledgments}

The author is grateful to the members of the Department of Anesthesiology, the Ninth People's 
Hospital, School of Medicine, Shanghai Jiaotong University, for their constant laboratory assistance.

\section{References}

1. Bekker A, Haile M, Kline R, et al. The effect of intraoperative infusion of dexmedetomidine on the quality of recovery after major spinal surgery. J Neurosurg Anesthesiol 2013; 25: 16-24.

2. Hayashi Y, Maze M. Alpha 2 adrenoceptor agonists and anaesthesia. Br J Anaesth 1993; 71: 108-18.

3. Sakurai Y, Obata T, Odaka A, Terui K, Tamura M, Miyao H. Buccal administration of dexmedetomidine as a preanesthetic in children. J Anesth 2010; 24: 49-53.

4. Taiji K. Dexmedetomidine hydrochloride (Precedex), a new sedative in intensive care, its pharmacological characteristics and clinical study result. Folia Pharmacologica Japonica 2004; 124: 171.

5. Li M, Zhang LP, Wu XM. Advance in search for clinical use of dexmedetomidine in anesthesiology. Chin J Clin Pharmacol 2007; 23: 466.

6. Lin L, Guo X, Zhang MZ, Qu CJ, Sun Y, Bai J. Pharmacokinetics of dexmedetomidine in Chinese postsurgical intensive care unit patients. Acta Anaesthesiol Scand 2011; 55: 359-67.

7. Afonso J, Reis F. Dexmedetomidine: current role in anesthesia and intensive care. Rev Bras Anestesiol 2012; 62 118-33.

8. Khan ZP, Ferguson CN, Jones RM. Alpha-2 and imidazoline receptor agonists. Their pharmacology and therapeutic role. Anaesthesia 1999; 54: 146-65.

9. Farag E, Argalious M, Abd-Elsayed A, Ebrahim Z, Doyle DJ. The use of dexmedetomidine in anesthesia and intensive care: a review. Curr Pharm Des 2012; 18: 6257-65.

10. Tan JA, Ho KM. Use of dexmedetomidine as a sedative and analgesic agent in critically ill adult patients: a meta-analysis. Intensive Care Med 2010; 36: 926-39.

11. Biccard BM, Goga S, de Beurs J. Dexmedetomidine and cardiac protection for non-cardiac surgery: a meta-analysis of randomised controlled trials. Anaesthesia 2008; 63: 4-14.

12. Isik B, Arslan M, Tunga AD, Kurtipek O. Dexmedetomidine decreases emergence agitation in pediatric patients after sevoflurane anesthesia without surgery. Paediatr Anaesth 2006; 16: 748-53.

13. Gurbet A, Basagan-Mogol E, Turker G, Ugun F, Kaya FN Ozcan B. Intraoperative infusion of dexmedetomidine reduces perioperative analgesic requirements. Can J Anaesth 2006; 53: 646-52

14. Guler G, Akin A, Tosun Z, Ors S, Esmaoglu A, Boyaci A. Single-dose dexmedetomidine reduces agitation and provides smooth extubation after pediatric adenotonsillectomy. Paediatr Anaesth 2005; 15: 762-6.

15. Lawrence CJ, De Lange S. Effects of a single pre-operative dexmedetomidine dose on isoflurane requirements and peri-operative haemodynamic stability. Anaesthesia 1997; 52: 736-44.

16. Jaakola ML, Ali-Melkkila T, Kanto J, Kallio A, Scheinin H, Scheinin M. Dexmedetomidine reduces intraocular pressure, intubation responses and anaesthetic requirements in patients undergoing ophthalmic surgery. $\mathrm{Br}$ J Anaesth 1992; 68: 570-5.

17. Jalonen J, Hynynen M, Kuitunen A, et al. Dexmedetomidine as an anesthetic adjunct in coronary artery bypass grafting. Anesthesiology 1997; 86: 331-45.
18. Shukry M, Clyde MC, Kalarickal PL, Ramadhyani U. Does dexmedetomidine prevent emergence delirium in children after sevoflurane-based general anesthesia? Paediatr Anaesth 2005; 15: 1098-104.

19. Yildiz M, Tavlan A, Tuncer S, Reisli R, Yosunkaya A, Otelcioglu S. Effect of dexmedetomidine on haemodynamic responses to laryngoscopy and intubation: perioperative haemodynamics and anaesthetic requirements. Drugs R D 2006; 7: 43-52.

20. Talke P, Chen R, Thomas B, et al. The hemodynamic and adrenergic effects of perioperative dexmedetomidine infusion after vascular surgery. Anesth Analg 2000; 90: 834-9.

21. Ozkose Z, Demir FS, Pampal K, Yardim S. Hemodynamic and anesthetic advantages of dexmedetomidine, an alpha 2-agonist, for surgery in prone position. Tohoku J Exp Med 2006; 210: 153-60.

22. Menda F, Koner O, Sayin M, Ture H, Imer P, Aykac B. Dexmedetomidine as an adjunct to anesthetic induction to attenuate hemodynamic response to endotracheal intubation in patients undergoing fast-track CABG. Ann Card Anaesth 2010; 13: 16-21.

23. Soliman RN, Hassan AR, Rashwan AM, Omar AM. Prospective, randomized controlled study to assess the role of dexmedetomidine in patients with supratentorial tumors undergoing craniotomy under general anesthesia. Middle East J Anesthesiol 2011; 21: 23-33.

24. Bajwa SJ, Gupta S, Kaur J, Singh A, Parmar S. Reduction in the incidence of shivering with perioperative dexmedetomidine: a randomized prospective study. J Anaesthesiol Clin Pharmacol 2012; 28: 86-91.

25. Aho M, Scheinin M, Lehtinen AM, Erkola O, Vuorinen J, Korttila K. Intramuscularly administered dexmedetomidine attenuates hemodynamic and stress hormone responses to gynecologic laparoscopy. Anesth Analg 1992; 75: 932-9.

26. Bicer C, Esmaoglu A, Akin A, Boyaci A. Dexmedetomidine and meperidine prevent postanaesthetic shivering. Eur J Anaesthesiol 2006; 23: 149-53.

27. Aho M, Erkola O, Kallio A, Scheinin H, Korttila K. Dexmedetomidine infusion for maintenance of anesthesia in patients undergoing abdominal hysterectomy. Anesth Analg 1992; 75: 940-6.

28. Talke P, Li J, Jain U, et al. Effects of perioperative dexmedetomidine infusion in patients undergoing vascular surgery. The Study of Perioperative Ischemia Research Group. Anesthesiology 1995; 82: 620-33.

29. Massad IM, Mohsen WA, Basha AS, Al-Zaben KR, Al-Mustafa MM, Alghanem SM. A balanced anesthesia with dexmedetomidine decreases postoperative nausea and vomiting after laparoscopic surgery. Saudi Med J 2009; 30: 1537-41.

30. Mukhtar AM, Obayah EM, Hassona AM. The use of dexmedetomidine in pediatric cardiac surgery. Anesth Analg 2006; 103: 52-6.

31. But AK, Ozgul U, Erdil F, et al. The effects of pre-operative dexmedetomidine infusion on hemodynamics in patients with pulmonary hypertension undergoing mitral valve replacement surgery. Acta Anaesthesiol Scand 2006; 50: 1207-12.

32. Cheung CW, Ying CL, Chiu WK, Wong GT, Ng KF, Irwin MG. A comparison of dexmedetomidine and midazolam for sedation in third molar surgery. Anaesthesia 2007; 62: 1132-8. 